\title{
WHITNEY CONTINUA OF CURVES
}

\author{
HISAO KATO
}

\begin{abstract}
In this paper, we prove several theorems relating shape properties of Whitney continua of curves. In particular, we investigate the fundamental dimension and the shape type of Whitney continua of curves.
\end{abstract}

1. Introduction. By a continuum, we mean a compact connected metric space. Let $X$ be a continuum. Then the hyperspace $C(X)$ of subcontinua of $X$ is metrized with the Hausdorff metric (e.g., see [15]). In [27], Whitney showed that for any continuum $X$ there exists a map $\omega: C(X) \rightarrow[0, \omega(X)]$ satisfying

(1) $\omega(\{x\})=0$ for every $x \in X$, and

(2) if $A, B \in C(X)$ and $A \subsetneq B$, then $\omega(A)<\omega(B)$.

Any such map is called a Whitney map. We may think of the map $\omega$ as measuring the size of a continuum. It is well known that every Whitney map $\omega$ is monotone, i.e., $\omega^{-1}(t)$ is a continuum for $0 \leq t \leq \omega(X)$. The continua $\omega^{-1}(t)(0 \leq t<$ $\omega(X))$ are called Whitney continua. A topological property $\mathrm{P}$ is called a Whitney property if whenever $X$ has property $\mathrm{P}$, so does every Whitney continua $\omega^{-1}(t)$ $(0 \leq t<\omega(X))$ for any Whitney map $\omega: C(X) \rightarrow[0, \omega(X)]$. It has been shown that several geometric properties are not Whitney properties for $n$-dimensional continua $(n \geq 2)$. For example, in [17], Petrus showed that the property of being (a) an AR or (b) an FAR is not a Whitney property, more precisely, there is a Whitney map $\omega: C(D) \rightarrow[0, \omega(D)]$ such that $D$ is a disk and, for some $0<t<\omega(D), \omega^{-1}(t)$ is not an FAR, in fact, $\omega^{-1}(t)$ is not even an FANR (see [8, (1.12)]). But, for the case of curves (=1-dimensional continua) there are many geometric properties which are Whitney properties (see References). In partricular, Whitney continua of chainable continua and circle-like continua have been studied by many authors (see References). Krasinkiewicz [10] proved that the property of being (a) chainable or (b) proper circle-like is a Whitney property. Also, he proved that if $X$ is a circlelike continuum, then $\operatorname{Sh} \omega^{-1}(t)=\operatorname{Sh} X$ for any Whitney map $\omega$ for $C(X)$ and $\mathbf{0} \leq t<\omega(X)$ (see [11]). In [24], Rogers showed that for any continuum $X$ and any Whitney map $\omega$ for $C(X)$, there is an induced injection $r^{*}: H^{1}\left(\omega^{-1}(t)\right) \rightarrow H^{1}(X)$ for $0 \leq t \leq \omega(X)$. By using this result, he investigated Whitney continua of curves (see $[\mathbf{2 3}, \mathbf{2 4}])$. In $[\mathbf{8}]$, the author proved that the property of being an FAR is a Whitney property for curves.

The aim of this paper is to investigate several shape properties of Whitney continua of curves. In $\S 2$, we study the fundamental properties of Whitney continua of graphs. In particular, we define an index $n(G)$ for a graph $G$ and we prove

Received by the editors July 1, 1985 and, in revised form, March 21, 1986.

1980 Mathematics Subject Classification (1985 Revision). Primary 54B20, 54F43; Secondary 54B25, 54F40.

Key words and phrases. Curve, inverse limit, hyperspace, Whitney map, Whitney continua, FANR, movable, (strongly) winding curve, $\theta(m)$-curve, tree-like, circle-like. 
that if $n(G) \leq m$, then $\mathrm{Fd} \omega^{-1}(t) \leq m-1$ for any Whitney map $\omega$ for $C(G)$ and $0 \leq t \leq \omega(G)$. In $\S 3$, we define natural shape morphisms between Whitney continua, and by using the shape morphisms we investigate shape properties of Whitney continua of curves. We prove that if $X$ is a strongly winding curve, then $\operatorname{Sh} \omega^{-1}(t)=\operatorname{Sh} X$ for any Whitney map $\omega$ for $C(X)$ and $0 \leq t<\omega(X)$. Every tree-like continuum and every circle-like continuum are strongly winding curves. In $\S 4$, by using the technique of Rogers [24, Theorem 3], we prove that if $X$ is a $\theta(m)$-curve and each proper subcontinuum of $X$ is tree-like, then $\operatorname{Sh} \omega^{-1}(t)=\operatorname{Sh} X$ for any Whitney map $\omega$ for $C(X)$ and $0 \leq t<\omega(X)$.

We refer readers to [9 and $\mathbf{1 5}]$ for hyperspace theory, and to [1 and 13] for shape theory.

2. Whitney continua of graphs. In [3 and 4], Duda described and analyzed polyhedral models for hyperspaces of graphs. We assume that the word graph means a finite connected 1-dimensional polyhedron. In this section, we investigate fundamental properties of Whitney continua of graphs. Let $G$ be a graph with a triangulation $T$. For any points $x, y$ of $G$, which belong to 1 -simplex $\left\langle V_{0}, V_{1}\right\rangle \in T$, define $d(x, y)=\left|t-t^{\prime}\right|$, where $x=t V_{0}+(1-t) V_{1}, y=t^{\prime} V_{0}+\left(1-t^{\prime}\right) V_{1}$. Let $A$ be a subcontinuum of $G$. For $x, y \in A$ we define a metric $d_{A}$ on $A$ by

$$
d_{A}(x, y)=\inf \left\{\sum_{i=1}^{m} d\left(x_{i}, x_{i+1}\right) \mid x_{0}=x, x_{m+1}=y, x_{i} \text { and } x_{i+1}(0 \leq i \leq m)\right.
$$

belong to the 1-simplex $\sigma$ of $T$ such that the segment from

$$
\left.x_{i} \text { to } x_{i+1} \text { in } \sigma \text { is contained in } A \text { for } 0 \leq i \leq m\right\} \text {. }
$$

Note that for $x_{n}, y_{n} \in A_{n} \in C(G)$ with $\lim _{n \rightarrow \infty} x_{n}=x, \lim _{n \rightarrow \infty} y_{n}=y$, and $\lim _{n \rightarrow \infty} A_{n}=A$, we cannot conclude that $d_{A}(x, y)=\lim _{n \rightarrow \infty} d_{A_{n}}\left(x_{n}, y_{n}\right)$. But it is easily seen that if $A$ contains no simple closed curve ( $=$ a tree), we can conclude that $d_{A}(x, y)=\lim _{n \rightarrow \infty} d_{A_{n}}\left(x_{n}, y_{n}\right)$. Then we have

(2.1) PROPOSITION. (1) Let $G$ be a graph which contains a simple closed curve and let $\omega$ be any Whitney map for $C(G)$. Let $t_{0}=\min \{\omega(S) \mid S$ is a simple closed curve in $G\}$. Then there is a homotopy $H: \omega^{-1}\left(\left[0, t_{0}\right)\right) \times I \rightarrow \omega^{-1}\left(\left[0, t_{0}\right)\right)$ such that

(a) $H(\{x\} \times I)=\{x\}$ for each $x \in G$,

(b) $H(A, 0)=A, H(A, 1) \in w^{-1}(0)$ for each $A \in \omega^{-1}\left(\left[0, t_{0}\right)\right)$, and

(c) if $s<s^{\prime}$, then $H(A, s) \supsetneqq H\left(A, s^{\prime}\right)$ for each $A \in \omega^{-1}\left(\left(0, t_{0}\right)\right)$.

(2) $[\mathbf{6},(2.17)$ or 17, Proposition 12] Let $G$ be a tree and let $\omega$ be any Whitney map for $C(G)$. Then there is a homotopy $H: C(G) \times I \rightarrow C(G)$ such that

$\left(\mathrm{a}^{\prime}\right) H(\{x\} \times I)=\{x\}$ for each $x \in G$,

( $\left.\mathrm{b}^{\prime}\right) H(A, 0)=A, H(A, 1) \in w^{-1}(0)$ for each $A \in C(G)$, and

( $\left.\mathrm{c}^{\prime}\right)$ if $s<s^{\prime}$, then $H(A, s) \supsetneqq H\left(A, s^{\prime}\right)$ for each $A \in \omega^{-1}((0, \omega(G)])$.

ProOf. We shall prove (1). Since $A$ contains no simple closed curve for each $A \in$ $\omega^{-1}\left(\left[0, t_{0}\right)\right)$, we can choose an open covering $\mathfrak{U}=\left\{\left\langle U_{\lambda_{1}}, U_{\lambda_{2}}, \ldots, U_{\lambda_{k}}\right\rangle \mid\left(\lambda_{1}, \lambda_{2}, \ldots\right.\right.$, $\left.\left.\lambda_{k}\right) \in J\right\}$ of $\omega^{-1}\left(\left[0, t_{0}\right)\right)$ such that $\bigcup_{j=1}^{k} \bar{U}_{\lambda_{j}}$ is a subcontinuum of $G$ and contains no simple closed curve for each $\left\langle U_{\lambda_{1}}, U_{\lambda_{2}}, \ldots, U_{\lambda_{k}}\right\rangle \in \mathfrak{U}$, where $U_{\lambda_{j}}$ is a connected 
open subset of $G$ and

$$
\begin{aligned}
& \left\langle U_{\lambda_{1}}, U_{\lambda_{2}}, \ldots, U_{\lambda_{k}}\right\rangle \\
& \quad=\left\{A \in \omega^{-1}\left(\left[0, t_{0}\right)\right) \mid A \cap U_{\lambda_{j}} \neq \varnothing(j=1,2, \ldots, k) \text { and } A \subset \bigcup_{j=1}^{k} U_{\lambda_{j}}\right\} .
\end{aligned}
$$

Choose a locally finite closed covering $\mathfrak{V}=\{\mathfrak{A}\}$ of $\omega^{-1}\left(\left[0, t_{0}\right)\right)$ which is a refinement of $\mathfrak{U}$. Now, we shall prove that if $\mathfrak{A} \in \mathfrak{V}$ and if $\mathfrak{B}$ is a closed subset of $\mathfrak{A}$ and $f: \mathfrak{B} \rightarrow G$ is a map such that $f(A) \in A$ for each $A \in \mathfrak{B}$, then there exists an extension $\tilde{f}: \mathfrak{A} \rightarrow G$ of $f$ such that $\tilde{f}(A) \in A$ for each $A \in \mathfrak{A}$. In fact, take a $\left\langle U_{\lambda_{1}}, U_{\lambda_{2}}, \ldots, U_{\lambda_{k}}\right\rangle \in \mathfrak{U}$ such that $\mathfrak{A} \subset\left\langle U_{\lambda_{1}}, U_{\lambda_{2}}, \ldots, U_{\lambda_{k}}\right\rangle$. Since $\bigcup_{j=1}^{k} \bar{U}_{\lambda_{j}}$ is an $\mathrm{AR}$, there is an extension $f^{\prime}: \mathfrak{A} \rightarrow \bigcup_{j=1}^{k} \bar{U}_{\lambda_{j}}$ of $f$ (note that $f(\mathfrak{B}) \subset \bigcup_{j=1}^{k} \bar{U}_{\lambda_{j}}$ ). Also, since $\bigcup_{j=1}^{k} \bar{U}_{\lambda_{j}}$ is a tree, for each $A \in \mathfrak{A}$, there is the unique point $\tilde{f}(A)$ of $A$ such that

$$
\left[f^{\prime}(A), \tilde{f}(A)\right] \cap A=\{\tilde{f}(A)\}
$$

where $\left[f^{\prime}(A), \tilde{f}(A)\right]$ denotes the unique arc from $f^{\prime}(A)$ to $\tilde{f}(A)$ in $\bigcup_{j=1}^{k} \bar{U}_{\lambda_{j}}$.

Clearly, the function $\tilde{f}$ is an extension of $f$ and it is continuous such that $\tilde{f}(A) \in$ $A$ for each $A \in \mathfrak{A}$. By using this fact, we can easily see that there is a map $h: \omega^{-1}\left(\left[0, t_{0}\right)\right) \rightarrow G$ such that $h(A) \in A$ for each $A \in \omega^{-1}\left(\left[0, t_{0}\right)\right)$.

Next, we shall define a homotopy $H: \omega^{-1}\left(\left[0, t_{0}\right)\right) \times I \rightarrow \omega^{-1}\left(\left[0, t_{0}\right)\right)$ satisfying the desired conditions. Let $A \in \omega^{-1}\left(\left[0, t_{0}\right)\right)$. Define a homotopy $H_{A}: A \times I \rightarrow A$ by $H_{A}(x, s)=y$ for $x \in A, s \in I$, where $y \in[x, h(A)]$ and $d_{A}(x, y)=s \cdot d_{A}(x, h(A))$. Then $H_{A}(x, 0)=x$ and $H_{A}(x, 1)=h(A)$ for $x \in A$. Also, define a function $H: \omega^{-1}\left(\left[0, t_{0}\right)\right) \times I \rightarrow \omega^{-1}\left(\left[0, t_{0}\right)\right)$ by

$$
H(A, s)=\left\{H_{A}(x, s) \mid x \in A\right\} .
$$

It is easily seen that $H$ is continuous and $H$ has the desired conditions. This completes the proof.

(2.2) REMARK. In the statement of (1) of (2.1), there is no homotopy $H: \omega^{-1}\left(\left[0, t_{0}\right]\right) \times I \rightarrow \omega^{-1}\left(\left[0, t_{0}\right]\right)$ satisfying the conditions (a), (b), and (c) of (1). Suppose, on the contrary, that such a homotopy $H$ exists. Let $S$ be a simple closed curve such that $\omega(S)=t_{0}$. By (c), $H(C(S) \times I) \subset C(S)$. By (a) and (b), $F_{1}(S)=\{\{x\} \mid x \in S\} \cong S$ is a strong deformation retract of $C(S)$. Since $C(S)$ is a disk, this implies a contradiction.

(2.3) Proposition. Let $G$ be a graph and let $\omega$ be any Whitney map for $C(G)$. If $G$ contains a simple closed curve, assume $t_{0}=\min \{\omega(S) \mid S$ is a simple closed curve in $G\}$. If $G$ is a tree, assume that $t_{0}=w(G)$. Then $\omega^{-1}(t)\left(0 \leq t<t_{0}\right)$ is homotopy equivalent to $G$, i.e., $\omega^{-1}(t) \simeq G$.

Proof. We shall prove only the case that $G$ contains a simple closed curve. The case that $G$ is a tree has been proved in [17]. Since $G$ is a Peano continuum, there is a convex metric $\rho$ on $G$. Define a homotopy $K: C(G) \times I \rightarrow C(G)$ by $K(A, s)=$ $B(A, \alpha(A, s))$, where $\alpha(A, s)$ is the positive number such that $B(A, \alpha(A, s))=$ $\{y \in G \mid \rho(A, y) \leq \alpha(A, s)\}$ and $\omega(B(A, \alpha(A, s)))=(1-s) \cdot \omega(A)+s \cdot \omega(G)$. Let $0 \leq t<t_{0}$. Define a map $f_{0 t}: G \rightarrow \omega^{-1}(t)$ by $f_{0 t}(x)=K(\{x\}, \beta(x))$, where 
$\omega(K(\{x\}, \beta(x)))=t$. By using the homotopy $H$ as in (2.1) and the homotopy $K$, we can easily see that $f_{0 t}$ is a homotopy equivalence (cf. $[\mathbf{7},(2.5)]$ ). Hence $\omega^{-1}(t) \simeq G$.

(2.4) Proposition. Let $G$ be a graph. Then for any Whitney map $\omega$ for $C(G), \omega^{-1}(t)$ is a polyhedron for each $0 \leq t \leq \omega(G)$.

SKETCH OF THE PROOF. The proof is very similar to the proof of $[\mathbf{3} ; \mathbf{4},(6.2)$ and (6.4)]. Let $A \subset B$ be a pair as in [3, p. 270] and let $\mathfrak{M}_{A \subset B}$ be the same family of subcontinua of $X$ as in $\left[3\right.$, p. 270]. Set $\mathfrak{M}_{A \subset B}(t)=\mathfrak{M}_{A \subset B} \cap \omega^{-1}(t)$. By similar arguments as in the proofs of $[3,(5.2)$ and $4,(6.2)]$, we obtain the following (see [4, (6.2)]): Let $A \subset B$ and $A^{\prime} \subset B^{\prime}$ be two distinct pairs of $G$. Then $\mathfrak{M}_{A \subset B}(t)$ and $\mathfrak{M}_{A^{\prime} \subset B^{\prime}}(t)$ are balls. Moreover, if $\mathfrak{M}_{A \subset B}(t)$ and $\mathfrak{M}_{A^{\prime} \subset B^{\prime}}(t)$ meet, then their common part either is equal to a ball or consists of finitely many disjoint balls, each lying on the surfaces of both $\mathfrak{M}_{A \subset B}(t)$ and $\mathfrak{M}_{A^{\prime} \subset B^{\prime}}(t)$. Hence we see that $\omega^{-1}(t)$ is a polyhedron.

Next, we need the following result of Lynch [14]. The author wishes to thank Professor D. Curtis for informing him of the result.

(2.5) (M. LYNCH [14]). Let $X$ be any continuum and $A \in C(X)$. Then for any Whitney map $\omega$ for $C(X)$, the set

$$
C_{A}(X, \omega, t)=\left\{B \in \omega^{-1}(t) \mid B \supset A\right\}
$$

is an $A R$ for $0 \leq t \leq \omega(X)$, where $\omega(A) \leq t$.

The author wishes to thank Mr. K. Kawamura for pointing out the graph $G(m)$ as in the following proposition.

(2.6) Proposition. Let $G=G(m)=\bigcup_{i=1}^{m} A_{i}(m \geq 2)$, where each $A_{i}$ $(i=1,2, \ldots, m)$ is an arc from $V_{1}$ to $V_{2}$ and $A_{i} \neq A_{j}(i \neq j)$. Assume that $A_{i} \cap A_{j}=\left\{V_{1}, V_{2}\right\}(i \neq j)$. Let $\omega$ be any Whitney map for $C(G)$ and let

$$
t_{0}=\max \left\{w\left(A_{1} \cup A_{2} \cup \cdots \cup A_{i-1} \cup A_{i+1} \cup \cdots \cup A_{m}\right) \mid i=1,2, \ldots, m\right\}<\omega(G) .
$$

Then $\omega^{-1}(t) \simeq S^{m-1}$ for $t_{0} \leq t<\omega(G)$, where $S^{m-1}$ denotes the $(m-1)$-sphere.

PROOF. Let $t_{0} \leq t<\omega(G)$. Since $t \geq \omega\left(A_{1} \cup A_{2} \cup \cdots \cup A_{i-1} \cup A_{i+1} \cup \cdots \cup A_{m}\right)$ and $G-\left\{V_{1}, V_{2}\right\}$ is not connected, we have

(1) $\omega^{-1}(t)=C_{V_{1}}(G, \omega, t) \cup C_{V_{2}}(G, \omega, t)$, which is the union of two ARs (see $(2.5))$.

Also, we have

(2) $C_{V_{1}}(G, \omega, t) \cap C_{V_{2}}(G, \omega, t)=\bigcup_{i=1}^{m} C_{A_{i}}(G, \omega, t)$, which is the union of $m$ ARs (see $(2.5))$.

Now, we shall prove that $C_{V_{1}}(G, \omega, t) \cap C_{V_{2}}(G, \omega, t) \simeq S^{m-2}$. Note that

(3) $\bigcap_{j \in J} C_{A_{j}}(G, \omega, t)=C_{\left(\bigcup_{j \in J} A_{j}\right)}(G, \omega, t)$ for each subset $J$ of $\{1,2, \ldots, m\}$.

By (3) and the definition of $t_{0}$, we have

(4) if $|J| \leq m-1$, then $\bigcap_{j \in J} C_{A_{j}}(G, \omega, t)$ in an AR (nonempty) and $\bigcap_{j=1}^{m} C_{A_{j}}(G, \omega, t)=C_{G}(G, \omega, t)=\varnothing$.

Let $\Delta$ be the $(m-1)$-simplex with vertices $a_{1}, a_{2}, \ldots, a_{m}$ and let $\partial \Delta$ denote the boundary of $\Delta$. Consider the barycentric subdivision $\operatorname{Sd} \partial \Delta$ and the decomposition $\left\{\operatorname{St}\left(a_{i} ; \operatorname{Sd} \partial \Delta\right) \mid i=1,2, \ldots, m\right\}$ of $\partial \Delta$ (see Figure 1 ). 


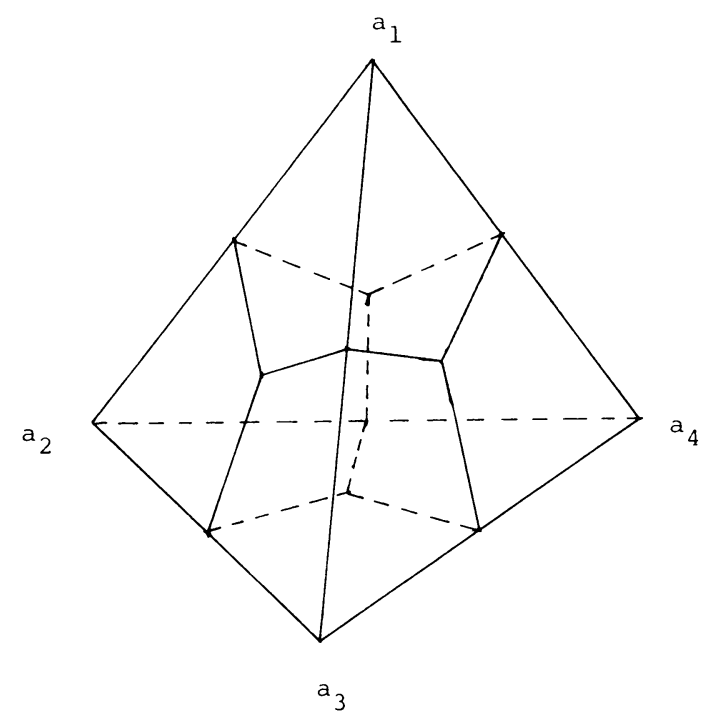

FIGURE 1

By (4), we can easily see that there exists a map $f: \partial \Delta \rightarrow C_{V_{1}}(G, \omega, t) \cap$ $C_{V_{2}}(G, \omega, t)$ such that

(5) $f\left(\bigcap_{j \in J} \operatorname{St}\left(a_{j} ; \operatorname{Sd} \partial \Delta\right)\right) \subset \bigcap_{j \in J} C_{A_{j}}(G, \omega, t)$ for each subset $J$ of $\{1,2, \ldots, m\}$.

Also, we have a map $g: C_{V_{1}}(G, \omega, t) \cap C_{V_{2}}(G, \omega, t) \rightarrow \partial \Delta$ such that

(6) $g\left(\bigcap_{j \in J} C_{A_{j}}(G, \omega, t)\right) \subset \bigcap_{j \in J} \operatorname{St}\left(a_{j} ; \operatorname{Sd} \partial \Delta\right)$ for each subset $J$ of $\{1,2, \ldots, m\}$.

Then we can easily see that $g f$ (resp. $f g$ ) is homotopic to the identity map on $\partial \Delta$ (resp. $C_{V_{1}}(G, \omega, t) \cap C_{V_{2}}(G, \omega, t)$ ), which implies that $C_{V_{1}}(G, \omega, t) \cap C_{V_{2}}(G, \omega, t) \simeq$ $S^{m-2}$. Let $S^{m-1}=D_{1} \cup D_{2}$, where $D_{i}(i=1,2)$ are $(m-1)$-balls such that $D_{1} \cap D_{2}$ is homeomorphic to $S^{m-2}$. As before, we have a map $h: D_{1} \cap D_{2} \rightarrow$ $C_{V_{1}}(G, \omega, t) \cap C_{V_{2}}(G, \omega, t)$ which is a homotopy equivalence. Since $C_{V_{i}}(G, \omega, t)$ is an $\mathrm{AR}$, there is an extension $\tilde{h}: S^{m-1} \rightarrow C_{V_{1}}(G, \omega, t) \cup C_{V_{2}}(G, \omega, t)=\omega^{-1}(t)$ of $h$ such that $\tilde{h}\left(D_{i}\right) \subset C_{V_{i}}(G, \omega, t)(i=1,2)$. Then we can easily see that $\tilde{h}: S^{m-1} \rightarrow \omega^{-1}(t)$ is a homotopy equivalence. Hence $\omega^{-1}(t) \simeq S^{m-1}$.

(2.7) Proposition. Let $G$ be a graph. If $G$ contains a subgraph $L$ which is homeomorphic to $G(m)$ as in (2.6), then there exist a Whitney map $\omega$ for $C(G)$ and a positive number $0<t<\omega(G)$ such that $\mathrm{Fd} \omega^{-1}(t) \geq m-1$ (see $[1, p .227$ or 13] for the definition of $\left.\mathrm{Fd} \omega^{-1}(t)\right)$.

Proof. By induction, we can easily see that there is a metric $\rho$ on $G$ and a retraction $r: G \rightarrow L$ such that $\rho(x, y) \geq \rho(r(x), r(y))$ for $x, y \in G$. Let $\omega$ be the Whitney map for $C(G)$ as is defined by $[\mathbf{2 7}]$ and the metric $\rho$. Let $r^{*}: C(G) \rightarrow$ $C(L)$ be the retraction of hyperspaces induced by $r$. Then we can easily see that $\omega\left(r^{*}(A)\right) \leq \omega(A)$ for each $A \in C(G)$. Hence $r^{*} \mid \omega^{-1}([0, t]): \omega^{-1}([0, t]) \rightarrow \omega_{L}^{-1}([0, t])$ is a retraction for any $0 \leq t \leq \omega(G)$, where $\omega_{L}=\omega \mid C(L)$. By $(2.6)$, there is $t>0$ such that $\omega_{L}^{-1}([0, t]) \simeq \omega_{L}^{-1}(t) \simeq S^{m-1}$. Hence $\mathrm{Fd} \omega^{-1}(t)=\mathrm{Fd} \omega^{-1}([0, t]) \geq m-1$. This completes the proof.

(2.8) EXAMPLE. Let $G=A_{1} \cup A_{2} \cup A_{3}$, where each $A_{i}$ is an arc from $V$ to $W$ and $A_{1} \cap A_{2} \cap A_{3}=A_{i} \cap A_{j}=\{V, W\}(i \neq j)$. Let $\omega$ be any Whitney map 
for $C(G)$. Set $t_{1}=\min \left\{\omega\left(A_{i}\right) \mid i=1,2,3\right\}, t_{2}=\min \left\{\omega\left(A_{i} \cup A_{j}\right) \mid i \neq j\right\}$, and $t_{3}=\max \left\{\omega\left(A_{i} \cup A_{j}\right) \mid i \neq j\right\}$. Then the Whitney continua $\omega^{-1}(t)$ are as follows:

(i) $t=0$ (see Figure 2).

(ii) $0<t<t_{1}$ (see Figure 3).

(iii) $t_{1} \leq t<t_{2}$ (see Figure 4).

(iv) $t_{2} \leq t<t_{3}$ (see Figure 5).

(v) $t_{3} \leq t<\omega(G)$ (see Figure 6).

(i) $t=0$

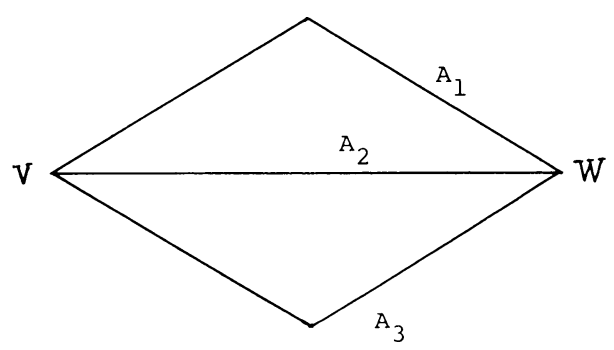

FIGURE 2

(ii) $0<t<t_{1}$

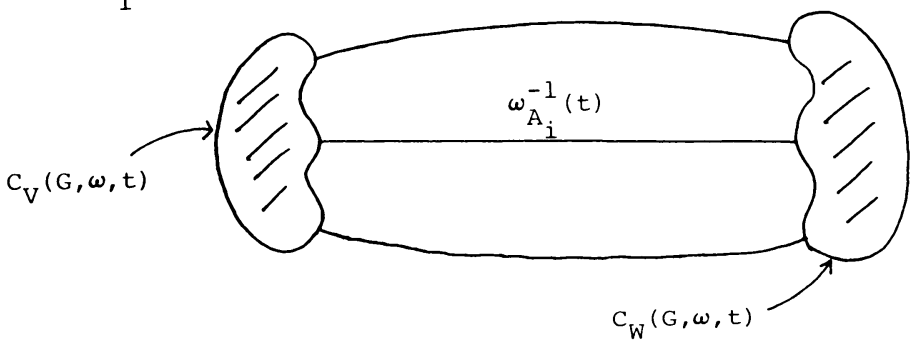

FIGURE 3

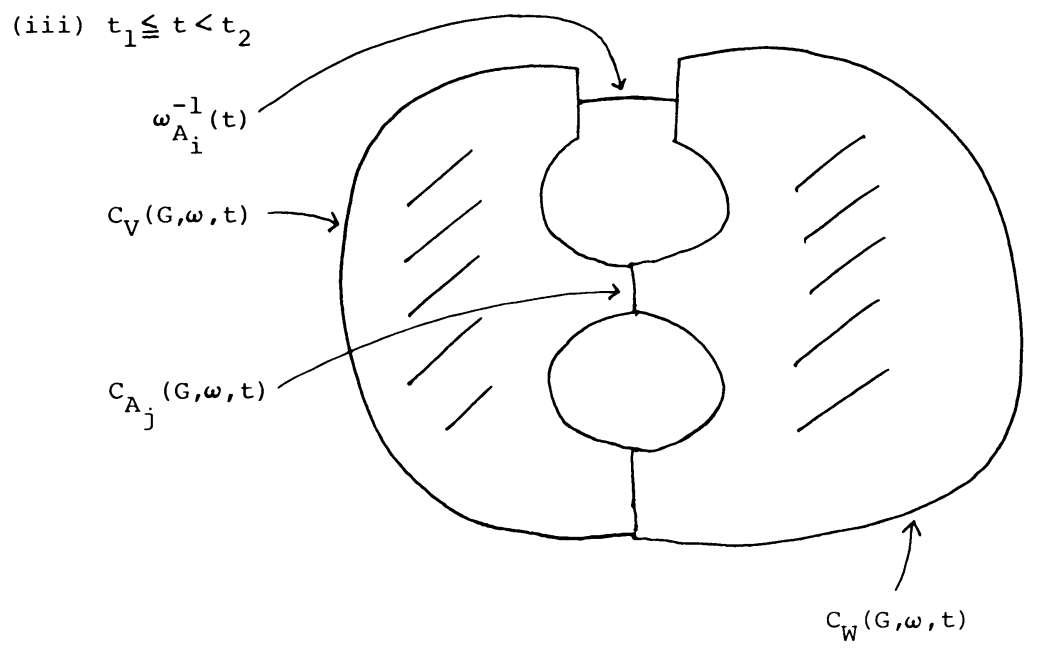

FIGURE 4 
(iv) $t_{2} \leqq t<t_{3}$

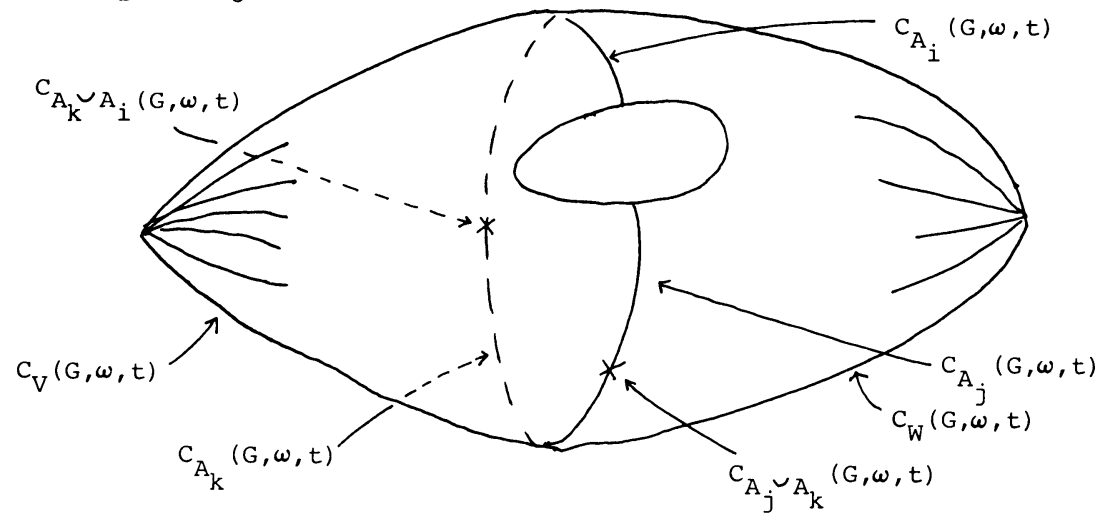

FIGURE 5

(V) $t_{3} \leqq t<\omega(G)$

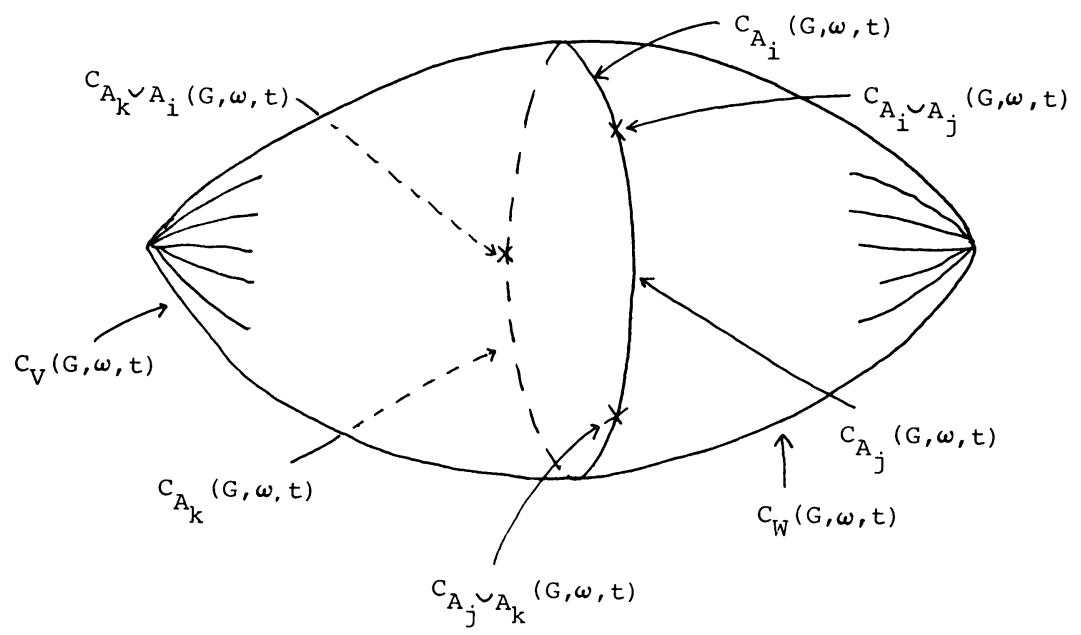

FIGURE 6

Let $G$ be a graph and let $e=\langle V, W\rangle$ be an edge of $G$. Consider the family $A(e)=\left\{A_{i}\right\}$ of arcs from $V$ to $W$ in $G$. Note that $e \in A(e)$. Set $n(e)=|A(e)|$, where $|A(e)|$ denotes the cardinal number of $A(e)$. Also, set $n(G)=\max \{n(e) \mid e$ is an edge of $G$ \}. Note that $n(G)$ is a topological invariant of $G$. Clearly, a graph $G$ is a tree if and only if $n(G) \leq 1$. Then we have the following

(2.9) THEOREM. Let $G$ be a graph and let $m=1,2, \ldots$ If $n(G) \leq m$, then, for any Whitney map $\omega$ for $C(G), \operatorname{Fd} \omega^{-1}(t) \leq m-1$ for $0 \leq t \leq \omega(G)$.

To prove (2.9), we need the following

(2.10) (S. NOWAK $[28,(4.1)])$. Let $X, Y$ be compacta. Then $\operatorname{Fd}(X \cup Y) \leq \max \{\operatorname{Fd}(X), \operatorname{Fd}(Y), \operatorname{Fd}(X \cap Y)+1\}$. 
ProOF OF (2.9). We shall prove the theorem by induction on the number $i$ of edges of $G$. Let $0<t<\omega(G)$. The statement is easily seen to be true for $i=1$. Also, the case $m=1$ is true (cf. (2.3)). We consider the case $m \geq 2$. Assume that it is true for $i \leq k$, and consider a graph $G$ with $(k+1)$ edges. Let $e=\langle V, W\rangle$ be an edge of $G$ such that $G-e$ is connected. Set $L=\overline{G-e}$. Note that $n(L) \leq m$. If $e \cap L=\{V\}$, then $\omega^{-1}(t)=\omega_{L}^{-1}(t) \cup \omega_{e}^{-1}(t) \cup C_{V}(G, \omega, t)$. By assumption, we can easily see that $\mathrm{Fd} \omega^{-1}(t)=\mathrm{Fd} \omega_{L}^{-1}(t) \leq m-1$, because $\omega_{e}^{-1}(t)$ and $C_{V}(G, \omega, t)$ are ARs. Now we assume that $e \cap L=\{V, W\}$. Then we have

$$
\omega^{-1}(t)=\omega_{L}^{-1}(t) \cap C_{V}(G, \omega, t) \cup C_{W}(G, \omega, t) \cup \omega_{e}^{-1}(t) .
$$

Consider the following cases (i) $\omega(e)>t$ and (ii) $\omega(e) \leq t$.

Case (i): $\omega(e)>t$. Note that $\omega_{e}^{-1}(t)$ is an arc. Then $C_{V}(G, \omega, t) \cap C_{W}(G, \omega, t)=$ $\bigcup_{j=1}^{s} C_{A_{i}}(G, \omega, t)$, where $A_{i}$ is an arc from $V$ to $W$ in $L$. Note that $s \leq m-$ 1. If $\omega(L) \leq t$, then $\omega^{-1}(t)=C_{V}(G, \omega, t) \cup C_{W}(G, \omega, t) \cup \omega_{e}^{-1}(t)$. Note that $\bigcap_{j=1}^{s} C_{A_{j}}(G, \omega, t)=C_{\bigcup_{j \in J} A_{j}}(G, \omega, t)$ is an AR for each subset $J$ of $\{1,2, \ldots, s\}$. Hence $C_{V}(G, \omega, t) \cap C_{W}(G, \omega, t)$ is an AR. Thus we can easily see that $\omega^{-1}(t) \simeq S^{1}$, which implies that $\operatorname{Fd} \omega^{-1}(t)=1 \leq m-1$. Assume that $\omega(L)>t$. Then $\omega_{L}^{-1}(t)$ is a strong deformation retract of $\omega_{L}^{-1}(t) \cup C_{V}(G, \omega, t) \cup C_{W}(G, \omega, t)$. By assumption, we have

$$
\operatorname{Fd}\left(\omega_{L}^{-1}(t) \cup C_{V}(G, \omega, t) \cup C_{W}(G, \omega, t)\right)=\mathrm{Fd} \omega_{L}^{-1}(t) \leq m-1 .
$$

Since $\left(\omega_{L}^{-1}(t) \cup C_{V}(G, \omega, t) \cup C_{W}(G, \omega, t)\right) \cap \omega_{e}^{-1}(t)$ consists of two points, by (2.10) we have $\mathrm{Fd} \omega^{-1}(t) \leq m-1$.

Case (ii): $\omega(e) \leq t$. Note that $\omega^{-1}(t)=\omega_{L}^{-1}(t) \cup C_{V}(G, \omega, t) \cup C_{W}(G, \omega, t)$. Since $C_{V}(G, \omega, t) \cap C_{W}(G, \omega, t)=\bigcup_{i=1}^{s} C_{A_{i}}(G, \omega, t) \cup C_{e}(G, \omega, t)$, by similar arguments as in the proof of (2.6) we can conclude that $C_{V}(G, \omega, t) \cap C_{W}(G, \omega, t)$ is homotopy equivalent to a $(m-2)$-dimensional polyhedron $P$. Also, $C_{V}(G, \omega, t) \cup$ $C_{W}(G, \omega, t)$ is homotopy equivalent to $\Sigma P$, where $\Sigma P$ denotes the suspension of $P$. Hence $\operatorname{Fd}\left(C_{V}(G, \omega, t) \cup C_{W}(G, \omega, t)\right) \leq m-1$. Note that $\omega_{L}^{-1}(t) \cap\left(C_{V}(G, \omega, t) \cup\right.$ $\left.C_{W}(G, \omega, t)\right)=C_{V}\left(L, \omega_{L}, t\right) \cup C_{W}\left(L, \omega_{L}, t\right)$. Since $C_{V}\left(L, \omega_{L}, t\right) \cap C_{W}\left(L, \omega_{L}, t\right)=$ $\bigcup_{i=1}^{s} C_{A_{i}}\left(L, \omega_{L}, t\right)$ and $s \leq m-1, C_{V}\left(L, \omega_{L}, t\right) \cup C_{W}\left(L, \omega_{L}, t\right)$ is homotopy equivalent to an (m-2)-dimensional polyhedron. Hence $\mathrm{Fd}\left(C_{V}\left(L, \omega_{L}, t\right) \cup C_{W}\left(L, \omega_{L}, t\right)\right) \leq$ $m-2$. By (2.10), we can conclude that $\mathrm{Fd} \omega^{-1}(t) \leq m-1$.

(2.11) EXAMPLE. Let $C$ be the Cantor set in the real line $E$, i.e., $C=\{x=$ $\sum_{i=1}^{\infty} a_{i} / 3^{i} \mid a_{i}=0$ or $\left.2(i=1,2, \ldots)\right\} \subset E=E \times\{0\} \subset E^{2}$. Consider the points $p=(0,1), q=(0,-1)$ of the plane $E^{2}$, and the sets $A_{x}=[p, x] \cup[q, x] \subset E^{2}$ for each $x \in C$, where $[p, x]$ denotes the segment from $p$ to $x$ in $E^{2}$. Set $X=\Sigma C$, where $\Sigma C$ denotes the suspension of $C$ with vertices $p$ and $q$, i.e., $X=\bigcup_{x \in C} A_{x}$. Let $\omega$ be the Whitney map for $C(X)$ as defined by $[\mathbf{2 7}]$ and the Euclidean metric $\rho$. Let $A \in$ $C(X)$. For each $n \geq 2$, let $F_{n}(A)=\{K \subset A \mid K \neq \varnothing$ and the cardinality of $K \leq n\}$, define $\lambda_{n}: F_{n}(A) \rightarrow[0, \infty)$ by letting $\lambda_{n}\left(\left\{a_{1}, a_{2}, \ldots, a_{n}\right\}\right)=\min \left\{\rho\left(a_{i}, a_{j}\right) \mid i \neq j\right\}$ for each $\left\{a_{1}, a_{2}, \ldots, a_{n}\right\} \in F_{n}(A)$, and let $\omega_{n}(A)=\sup \lambda_{n}\left(F_{n}(A)\right)$. Then $\omega(A)=$ $\sum_{n=2}^{\infty} \omega_{n}(A) / 2^{n-1}$ (see [27]). Now, we shall show that $\mathrm{Fd} \omega^{-1}(t)=\infty$ for some $t$ $(0<t<\omega(X))$. First, we show that if $x_{i} \in C(i=1,2, \ldots, k)$ and $x_{i}<x<y$ $(x, y \in C)$, then $\omega\left(\bigcup_{i=1}^{k} A_{x_{i}} \cup A_{x}\right)<\omega\left(\bigcup_{i=1}^{k} A_{x_{i}} \cup A_{y}\right)$. In fact, since there is a homeomorphism $h: \bigcup_{i=1}^{k} A_{x_{i}} \cup A_{y} \rightarrow \bigcup_{i=1}^{k} A_{x_{i}} \cup A_{x}$ such that $\rho(a, b) \geq \rho(h(a), h(b))$ 
for each $a, b \in \bigcup_{i=1}^{k} A_{x_{i}} \cup A_{y}$, then $\omega_{n}\left(\bigcup_{i=1}^{k} A_{x_{i}} \cup A_{x}\right) \leq \omega_{n}\left(\bigcup_{i=1}^{k} A_{x_{i}} \cup A_{y}\right)$. Also, note that $\omega_{3}\left(\bigcup_{i=1}^{k} A_{x_{i}} \cup A_{x}\right)=\rho(p, x)<\rho(p, y)=\omega_{3}\left(\bigcup_{i=1}^{k} A_{x_{i}} \cup A_{y}\right)$. Hence $\omega\left(\bigcup_{i=1}^{k} A_{x_{i}} \cup A_{x}\right)<\omega\left(\bigcup_{i=1}^{k} A_{x_{i}} \cup A_{y}\right)$.

Set $s_{2}=\max \left\{\omega\left(A_{x_{2}(1)}\right), \omega\left(A_{x_{2}(2)}\right)\right\}$ and $t_{2}=\omega\left(A_{x_{2}(1)} \cup A_{x_{2}(2)}\right)$, where $x_{2}(1)=$ $0, x_{2}(2)=1$. Then $s_{2}<t_{2}$. By induction, for each $n=2,3, \ldots$, choose points $x_{n}(1), x_{n}(2), \ldots, x_{n}(n)$ of $C$ such that

(1) $x_{n}(1)<x_{n}(2)<\cdots<x_{n}(n), x_{n+1}(1)=x_{n}(1), \ldots, x_{n+1}(n-1)=x_{n}(n-1)$, and $x_{n}(n-1)<x_{n+1}(n)<x_{n+1}(n+1)<x_{n}(n)$,

(2) $s_{n}=\max \left\{\omega\left(A_{x_{n}(1)} \cup \cdots \cup A_{x_{n}(i-1)} \cup A_{x_{n}(i+1)} \cup \cdots \cup A_{x_{n}(n)}\right) \mid i=1,2, \ldots, n\right\}$, $t_{n}=\omega\left(\bigcup_{i=1}^{n} A_{x_{n}(i)}\right)$, and

(3) $s_{n-1}<s_{n}<t_{n}<t_{n-1}(n=3,4, \ldots)$.

Note that $x_{n}(1)=0$ for each $n=2,3, \ldots$ Let $t=\lim t_{n}$. We show that $\mathrm{Fd} \omega^{-1}(t)=\infty$. Since $s_{n}<t<t_{n}$ for each $n \geq 2$, by (2.6) $\mathrm{Fd} \omega_{Y_{n}}^{-1}(t)=n-1$, where $Y_{n}=\sum\left(\left\{x_{n}(1), x_{n}(2), \ldots, x_{n}(n)\right\}\right)=\bigcup_{i=1}^{n} A_{X_{n}(i)}$. Since $\omega_{Y_{n}}^{-1}(t)$ is an ANR, for some $t^{\prime}>t$ there exists a retraction $r: \omega_{Y_{n}}^{-1}\left(\left[0, t^{\prime}\right]\right) \rightarrow \omega_{Y_{n}}^{-1}([0, t])$. Let $r_{1}: X \rightarrow$ $Y_{n}$ be a retraction. Then we can choose a closed and open neighborhood $H_{n}$ of $\left\{x_{n}(1), x_{n}(2), \ldots, x_{n}(n)\right\}$ in $C$ such that $r_{1}^{*} \omega_{\sum H_{n}}^{-1}([0, t]) \subset \omega_{Y_{n}}^{-1}\left(\left[0, t^{\prime}\right]\right)$. Also, choose a retraction $r_{2}: X \rightarrow \sum H_{n}$ such that if $x, y \in X$, then $\rho\left(r_{2}(x), r_{2}(y)\right) \leq \rho(x, y)$. Then $r_{2}^{*}\left(\omega^{-1}([0, t])\right) \subset \omega_{\sum H_{n}}^{-1}([0, t])$. Since $r \circ r_{1}^{*} \circ r_{2}^{*}: \omega^{-1}([0, t]) \rightarrow \omega_{Y_{n}}^{-1}([0, t])$ is a retraction, we can conclude that

$$
\mathrm{Fd} \omega^{-1}(t)=\mathrm{Fd} \omega^{-1}([0, t]) \geq \operatorname{Fd} \omega_{Y_{n}}^{-1}([0, t])=\operatorname{Fd} \omega_{Y_{n}}^{-1}(t)=n-1
$$

(see [8, (1.3) Theorem] or (3.3)). Hence Fd $\omega^{-1}(t)=\infty$ (see Figure 7).

3. Shape of Whitney continua of curves. In this section, we define natural shape morphisms between Whitney continua by using inverse sequences. By using these shape morphisms, we investigate the shape of Whitney continua of curves.

In [8], the author defined shape morphisms between Whitney continua. In order to study Whitney continua of curves, we need another description of the shape morphisms by using inverse sequences of graphs, because the structures of Whitney continua of graphs are simple.

Let $\underline{X}=\left\{X_{n}, p_{n n+1}\right\}$ be an inverse sequence of continua and let $X=\underline{\lim } \underline{X}$. Then we have

(3.1) $[8,(4.2)]$. If $\omega: C(X) \rightarrow[0, \omega(X)]$ is any Whitney map for $C(X)$, then there exist Whitney maps $\omega_{n}: C\left(X_{n}\right) \rightarrow\left[0, \omega_{n}\left(X_{n}\right)\right]$ satisfying the conditions; for any $\varepsilon>0$, there is $n_{0}$ such that

$$
d\left(\omega, \omega_{n} p_{n}^{*}\right)=\sup \left\{\left|\omega(A)-\omega_{n} p_{n}^{*}(A)\right| \mid A \in C(X)\right\}<\varepsilon,
$$

and

$$
d\left(\omega_{m} p_{m n}^{*}, \omega_{n}\right)<\varepsilon \quad \text { for each } n \geq m \geq n_{0},
$$

where $p_{n}: X \rightarrow X_{n}$ denotes the natural projection.

(3.2) Proposition (CF. $[8,(4.1)])$. Let $\omega_{n}$ be Whitney maps for $C\left(X_{n}\right)$ as in (3.1). Then there are positive numbers $\alpha_{n}(n=1,2, \ldots)$ such that $\lim _{n \rightarrow \infty} \alpha_{n}=$ 


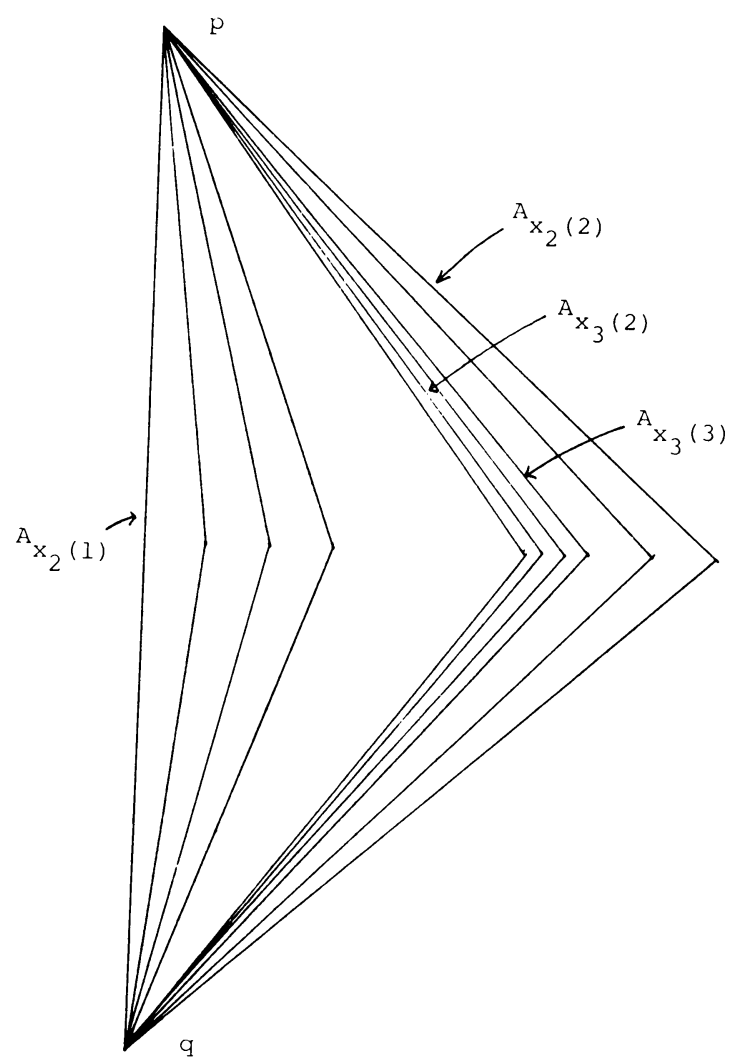

FIGURE 7

0 and $\omega^{-1}([s, t])=\underset{\lim }{\omega^{-1}}([s, t])$ for $0 \leq s \leq t \leq \omega(X)$, where $\underline{\omega^{-1}([s, t])}$ is an inverse sequence as follows:

$\omega_{n_{1}}^{-1}\left(\left[s-\alpha_{1}, t+\alpha_{1}\right]\right) \stackrel{p_{n_{1} n_{2}}^{*}}{\leftarrow} \omega_{n_{2}}^{-1}\left(\left[s-\alpha_{2}, t+\alpha_{2}\right]\right) \stackrel{p_{n_{2} n_{3}}^{*} \omega_{n_{3}}^{-1}\left(\left[s-\alpha_{3}, t+\alpha_{3}\right]\right)}{\leftarrow}$
$\left(n_{1}<n_{2}<n_{3}<\cdots\right)$.

PROOF. Let $\varepsilon_{1}>\varepsilon_{2}>\varepsilon_{3}>\cdots$ be a decreasing sequence of positive numbers such that $\sum \varepsilon_{i}<\infty$. By (3.1), there are Whitney maps $\omega_{n}$ for $C\left(X_{n}\right)$ such that

(1) $d\left(\omega, \omega_{n} p_{n}^{*}\right)<\varepsilon_{i}$ and

(2) $d\left(\omega_{m} p_{m n}^{*}, \omega_{n}\right)<\varepsilon_{i}$ for each $n \geq m \geq n_{i}$, where $n_{1}<n_{2}<\cdots$. Set $\alpha_{i}=\sum_{j=i}^{\infty} \varepsilon_{j}$. By (2), we have

(3) $p_{n_{i} n_{i+1}}^{*}\left(\omega_{n_{i+1}}^{-1}\left(\left[s-\alpha_{i+1}, t+\alpha_{i+1}\right]\right)\right) \subset \omega_{n_{i}}^{-1}\left(\left[s-\alpha_{i}, t+\alpha_{i}\right]\right)$.

Hence, we can consider the following inverse sequence $\omega^{-1}([s, t])$ :

$$
\omega_{n_{1}}^{-1}\left(\left[s-\alpha_{1}, t+\alpha_{1}\right]\right) \stackrel{p_{n_{1} n_{2}}^{*}}{\longleftarrow} \omega_{n_{2}}^{-1}\left(\left[s-\alpha_{2}, t+\alpha_{2}\right]\right) \stackrel{p_{n_{2} n_{3}}^{*}}{\longleftarrow} \omega_{n_{3}}^{-1}\left(\left[s-\alpha_{3}, t+\alpha_{3}\right]\right) \leftarrow .
$$

Let $A \in \omega^{-1}([s, t])$. By $(1)$, we have

$$
p_{n_{i}}^{*}(A) \subset \omega_{n_{i}}^{-1}\left(\left[s-\varepsilon_{i}, t+\varepsilon_{i}\right]\right) \subset \omega_{n_{i}}^{-1}\left(\left[s-\alpha_{i}, t+\alpha_{i}\right]\right) .
$$


Let $\left(A_{n_{i}}\right) \in \lim ^{\omega^{-1}([s, t])}$. Set $A=\lim _{\longleftarrow}\left\{A_{n_{i}},\left(p_{n_{i} n_{i+1}} \mid A_{n_{i+1}}\right)\right\}$. Then $A \in C(X)$. Also, we have

$$
s-\varepsilon_{i}-\alpha_{i} \leq \omega(A) \leq t+\varepsilon_{i}+\alpha_{i} \text { for each } i=1,2, \ldots .
$$

If $i \rightarrow \infty$, then $A \in \omega^{-1}([s, t])$. Thus $\omega^{-1}([s, t])=\lim \omega^{-1}([s, t])$.

Now, by using (3.2), we shall define desired shape morphisms between Whitney continua. Let $\underline{X}=\left\{X_{n}, p_{n n+1}\right\}$ be an inverse sequence of Peano continua and let $X=\lim _{\longleftarrow} \underline{X}$. Let $\omega$ be any Whitney map for $C(X)$. Then, by (3.2) there are Whitney maps $\omega_{n}$ for $C\left(X_{n}\right)$ and positive numbers $\alpha_{n}$ as in (3.2). Without loss of generality, we may assume that $n_{1}=1, n_{2}=2, \ldots, n_{i}=i, \ldots$ (see (3.2)). Since each $X_{n}$ is a Peano continuum, there is a convex metric $d_{n}$ on $X_{n}$. Define a homotopy $K_{n}: C\left(X_{n}\right) \times[0, \infty) \rightarrow C\left(X_{n}\right)$ (cf. the proof of (2.3)) by

$$
K_{n}(A, u)=\left\{x \in X_{n} \mid d_{n}(A, x) \leq u\right\} \quad \text { for } A \in C\left(X_{n}\right) \text { and } u \in[0, \infty) .
$$

Let $0 \leq s \leq t \leq \omega(X)$. Define a homotopy $F_{s t}^{n}: \omega_{n}^{-1}\left(\left[s-\alpha_{n}, t+\alpha_{n}\right]\right) \times I \rightarrow$ $\omega_{n}^{-1}\left(\left[s-\alpha_{n}, t+\alpha_{n}\right]\right)(n=1,2, \ldots)$ by

$$
F_{s t}^{n}(A, u)= \begin{cases}A, & \text { if } \omega(A) \geq t-\alpha_{n}, \\ K_{n}(A, \beta(A, u)), & \text { if } \omega(A) \leq t-\alpha_{n}\end{cases}
$$

where $\omega_{n}\left(K_{n}(A, \beta(A, u))\right)=u \cdot\left(t-\alpha_{n}\right)+(1-u) \omega(A)$. Consider the maps $r_{s t}^{n}: \omega_{n}^{-1}\left(\left[s-\alpha_{n}, t+\alpha_{n}\right]\right) \rightarrow \omega_{n}^{-1}\left(\left[t-\alpha_{n}, t+\alpha_{n}\right]\right)$ defined by

$$
r_{s t}^{n}=F_{s t}^{n} \mid \omega_{n}^{-1}\left(\left[s-\alpha_{n}, t+\alpha_{n}\right]\right) \times\{1\} .
$$

Then we have

(3.3) THEOREM. $\underline{r}_{s t}=\left\{r_{s t}^{n}\right\}_{n=1,2, \ldots}: \omega^{-1}([s, t]) \rightarrow \omega^{-1}(t)$ is a shape equivalence such that $\underline{r}_{s t} \underline{i}=\underline{1}_{\omega^{-1}(t)}$, where $\underline{i}: \omega^{-1}(t) \rightarrow \omega^{-1}([s, t])$ is the shape morphism induced by the inclusion map, and $\underline{1}_{\omega^{-1}(t)}$ denotes the identity morphism on $\omega^{-1}(t)$.

ProOF. First, we shall prove that

$$
\begin{aligned}
& r_{s t}^{n}\left(p_{n n+1}^{*} \mid \omega_{n+1}^{-1}\left(\left[s-\alpha_{n+1}, t+\alpha_{n+1}\right]\right)\right) \\
& \quad \simeq\left(p_{n n+1}^{*} \mid \omega_{n+1}^{-1}\left(\left[t-\alpha_{n+1}, t+\alpha_{n+1}\right]\right)\right) r_{s t}^{n+1} .
\end{aligned}
$$

Define a homotopy $R_{n}: \omega_{n+1}^{-1}\left(\left[s-\alpha_{n+1}, t+\alpha_{n+1}\right]\right) \times I \rightarrow \omega_{n}^{-1}\left(\left[t-\alpha_{n}, t+\alpha_{n}\right]\right)$ by

$$
R_{n}(A, u)= \begin{cases}r_{s t}^{n} F_{s t}^{n}\left(p_{n n+1}^{*}(A), 1-2 u\right), & \text { if } 0 \leq u \leq 1 / 2, \\ r_{s t}^{n} p_{n n+1}^{*} F_{s t}^{n+1}(A, 2 u-1), & \text { if } 1 / 2 \leq u \leq 1 .\end{cases}
$$

It is easily checked that $R_{n}(A, 0)=r_{s t}^{n} p_{n n+1}^{*}(A)$ and $R_{n}(A, 1)=p_{n n+1}^{*} r_{s t}^{n+1}(A)$ for $A \in \omega_{n+1}^{-1}\left(\left[s-\alpha_{n+1}, t+\alpha_{n+1}\right]\right)$, which implies (1). Since each $r_{s t}^{n}$ is a homotopy equivalence, $\underline{r}_{s t}$ is a shape equivalence. Also, since $r_{s t}^{n} \mid \omega_{n}^{-1}\left(\left[t-\alpha_{n}, t+\alpha_{n}\right]\right)=$ the identity map on $\omega_{n}^{-1}\left(\left[t-\alpha_{n}, t+\alpha_{n}\right]\right), \underline{r}_{s t} \underline{i}=\underline{1}_{\omega^{-1}(t)}$. This completes the proof.

Now, consider the shape morphisms $\underline{f}_{s t}=\left\{f_{s t}^{n}\right\}_{n=1,2, \ldots}: \omega^{-1}(s) \rightarrow \omega^{-1}(t)$ defined by $f_{s t}^{n}=r_{s t}^{n} \mid \omega^{-1}\left(\left[s-\alpha_{n}, s+\alpha_{n}\right]\right)$. Note that the shape morphisms $\underline{f}_{s t}$ is the same as in $\left[8,(1.13)\right.$ and (1.14)]. By using $\underline{f}_{s t}$, we investigate Whitney continua of curves.

A curve $X$ is said to be a winding curve (resp. strongly winding curve) if there is an inverse sequence $\underline{X}=\left\{G_{n}, p_{n n+1}\right\}$ of graphs such that $X=\underline{\lim } \underline{X}$ and $\underline{X}$ 
satisfies the following conditions: (*) If $S$ is a simple closed curve in $G_{n+1}$, then $p_{n n+1}(S)$ contains a simple closed curve (resp. (**) If $S$ is a simple closed curve in $G_{n+1}$, then $\left.p_{n n+1}(S)=G_{n}\right)$. Clearly, every tree-like continuum and every circlelike continuum are strongly winding curves. Also, the Case-Chamberlin curve [2] is a strongly winding curve.

Then we have the following

(3.4) THEOREM. Let $X$ be a winding curve and let $\omega$ be any Whitney map for $C(X)$. Then there is a positive number $t_{0}\left(t_{0}<\omega(X)\right)$ such that $\underline{f}_{0 t}: X \rightarrow \omega^{-1}(t)$ is a shape equivalence for $0 \leq t \leq t_{0}$, i.e., $\operatorname{Sh} \omega^{-1}(t)=\operatorname{Sh} X$.

PROOF. Let $\underline{X}=\left\{G_{n}, p_{n n+1}\right\}$ be an inverse sequence of graphs such that $X=\lim _{\longleftarrow} \underline{X}$ and $\underline{X}$ satisfies the conditions $(*)$. Consider the projection $p_{1}: X \rightarrow$ $G_{1}$. Take a positive number $t_{0}$ such that if $A \in \omega^{-1}\left(\left[0, t_{0}\right]\right), p_{1}^{*}(A)$ contains no simple closed curve. We shall prove that there is $n_{0}$ such that if $n \geq n_{0}$ for any $A \in \omega_{n}^{-1}\left(\left[0, t_{0}+\alpha_{n}\right]\right)$, then $A$ contains no simple closed curve in $G_{n}$, where $\omega_{n}$ is a Whitney map for $C\left(G_{n}\right)$ and $\alpha_{n}$ is a positive number as in $(3.2)(n=1,2, \ldots)$. Choose a neighborhood $\mathfrak{U}$ of $p_{1}^{*} \omega^{-1}\left(\left[0, t_{0}\right]\right)$ in $C\left(G_{1}\right)$ such that for any $A \in \mathfrak{U}, A$ contains no simple closed curve in $G_{1}$. By $(3.2), \omega^{-1}\left(\left[0, t_{0}\right]\right)=\lim \omega^{-1}\left(\left[0, t_{0}\right]\right)$, where $\omega^{-1}\left(\left[0, t_{0}\right]\right)=\left\{\omega_{n}^{-1}\left(\left[0, t_{0}+\alpha_{n}\right]\right), p_{n n+1}^{*} \mid \omega_{n+1}^{-1}\left(\left[0, t_{0}+\alpha_{n+1}\right]\right)\right\}$ is the inverse sequence as in (3.2). Consider the Freudenthal space $\sigma \omega^{-1}\left(\left[0, t_{0}\right]\right)$ of $\omega^{-1}\left(\left[0, t_{0}\right]\right)$, i.e., for an inverse sequence $\underline{Y}=\left\{Y_{n}, q_{n n+1}\right\}, \sigma \underline{Y}$ is the set $\lim _{\longleftarrow} \cup \bigcup_{n=1}^{\infty} Y_{n}$ with the topology defined by assuming the totality of the following sets; open subsets of the spaces $Y_{n}$, and sets of the form $q_{m}^{-1}(U) \cup \bigcup_{m \leq n} q_{m n}^{-1}(U)$, where $U$ is an open subset of $Y_{m}$. Define the map $p^{*}: \sigma \omega^{-1}\left(\left[0, t_{0}\right]\right) \rightarrow C\left(G_{1}\right)$ by $p^{*} \mid \omega^{-1}\left(\left[0, t_{0}\right]\right)=$ $p_{1}^{*} \mid \omega^{-1}\left(\left[0, t_{0}\right]\right)$ and $p^{*} \mid \omega_{n}^{-1}\left(\left[0, t_{0}+\alpha_{n}\right]\right) \overline{=p_{1 n}^{*} \mid \omega_{n}^{-1}}\left(\left[0, t_{0}+\alpha_{n}\right]\right)$ for $n \geq 1$. Note that $\lim _{n \rightarrow \infty} \omega_{n}^{-1}\left(\left[0, t_{0}+\alpha_{n}\right]\right)=\omega^{-1}\left(\left[0, t_{0}\right]\right)$ in $\sigma \omega^{-1}\left(\left[0, t_{0}\right]\right)$. Hence there is $n_{0}$ such that if $n \geq n_{0}$, then $p_{1 n}^{*} \omega_{n}^{-1}\left(\left[0, t_{0}+\alpha_{n}\right]\right) \subset \mathfrak{U}$. By $(*)$, we can easily see that $n_{0}$ is the desired positive integer. Let $0 \leq t \leq t_{0}$. Consider the shape morphism $\underline{f}_{0 t}: X \rightarrow \omega^{-1}(t)$ as before. Since $\omega_{n}^{-1}\left(t+\alpha_{n}\right)$ contains no simple closed curve $\left(n \geq n_{0}\right), f_{0 t}^{n}: G_{n} \rightarrow \omega_{n}^{-1}\left(\left[t-\alpha_{n}, t+\alpha_{n}\right]\right)$ is a homotopy equivalence (see the proof of (2.3)). Hence $\underline{f}_{0 t}$ is a shape eqivalence, i.e., $\operatorname{Sh} \omega^{-1}(t)=\operatorname{Sh} X$. This completes the proof.

(3.5) THEOREM. Let $X$ be a strongly winding curve and let $w$ be any Whitney map for $C(X)$. Then $f_{0 t}: X \rightarrow \omega^{-1}(t)$ is a shape equivalence for $0 \leq t<\omega(X)$, i.e., $\operatorname{Sh} \omega^{-1}(t)=\operatorname{Sh} X$.

ProOF. Let $\underline{X}=\left\{G_{n}, p_{n n+1}\right\}$ be an inverse sequence of graphs such that $X=\lim _{\longleftarrow} \underline{X}$ and $\underline{X}$ satisfies the condition $(* *)$. Let $\omega_{n}$ be the Whitney map for $C\left(G_{n}\right)$ and $\alpha_{n}$ be the positive number as in (3.2) $(n=1,2, \ldots)$. Let $0 \leq t<\omega(X)$. First, we shall prove that there is $n_{1}$ such that $p_{n_{1}}^{*}(A)$ contains no simple closed curve for each $A \in \omega^{-1}(t)$. In fact, for each $A \in \omega^{-1}(t)$, there is $n(A)$ such that $p_{n(A)}^{*}(A) \neq G_{n(A)}$. By $(* *), P_{n(A)+1}^{*}$ contains no simple closed curve. Choose a neighborhood $\mathfrak{U}(A)$ of $A$ in $\omega^{-1}(t)$ such that $p_{n(A)+1}^{*}(B)$ contains no simple closed curve for each $B \in \mathfrak{U}(A)$. Since $\omega^{-1}(t)$ is compact, there are finite points 
$A_{1}, A_{2}, \ldots, A_{m}$ of $\omega^{-1}(t)$ such that $\bigcup_{i=1}^{m} \mathfrak{U}\left(A_{i}\right)=\omega^{-1}(t)$. Set

$$
n_{1}=\max \left\{n\left(A_{1}\right)+1, n\left(A_{2}\right)+1, \ldots, n\left(A_{m}\right)+1\right\} .
$$

Then $p_{n_{1}}^{*}(A)$ contains no simple closed curve for each $A \in \omega^{-1}(t)$. By the same argument as in the proof of (3.4), there is $n_{0} \geq n_{1}$ such that if

$$
A \in \omega_{n}^{-1}\left(\left[t-\alpha_{n}, t+\alpha_{n}\right]\right) \quad\left(n \geq n_{0}\right),
$$

then $A$ contains no simple closed curve. Hence $f_{0 t}^{n}: X \rightarrow \omega_{n}^{-1}\left(\left[t-\alpha_{n}, t+\alpha_{n}\right]\right)$ $\left(n \geq n_{0}\right)$ are homotopy equivalence, which implies that $\underline{f}_{0 t}: X \rightarrow \omega^{-1}(t)$ is a shape equivalence, i.e., $\operatorname{Sh} \omega^{-1}(t)=\operatorname{Sh} X$. This completes the proof.

As corollaries of (3.5), we have

(3.6) Corollary (J. Krasinkiewicz $[\mathbf{1 1},(3.3)]$ ). Let $X$ be a circle-like continuum and let $\omega$ be any Whitney map for $C(X)$. Then $\operatorname{Sh} \omega^{-1}(t)=\operatorname{Sh} X$ for $0 \leq t<\omega(X)$.

(3.7) COROllary $[8,(4.1)]$. Let $X$ be a tree-like continuum and let $\omega$ be any Whitney map for $C(X)$. Then $\operatorname{Sh} \omega^{-1}(t)$ is trivial for $0 \leq t \leq \omega(X)$.

(3.8) Corollary. Let $X$ be the Case-Chamberlin curve and let $\omega$ be any Whitney map for $C(X)$. Then $\operatorname{Sh} \omega^{-1}(t)=\operatorname{Sh} X$ for $0 \leq t<\omega(X)$.

A curve $X$ is said to be a $\theta(m)$-curve $(m=1,2, \ldots)$ provided that there is an inverse sequence $\underline{X}=\left\{G_{i}, p_{i i+1}\right\}$ of graphs such that $n\left(G_{i}\right) \leq m$ for each $i$. Note that a curve $X$ is tree-like if and only if $X$ is a $\theta(1)$-curve. By (2.9) and (3.2), we have

(3.9) Proposition. Let $X$ be a curve and let $\omega$ be any Whitney map for $C(X)$. If $X$ is a $\theta(m)$-curve, then $\mathrm{Fd} \omega^{-1}(t) \leq m-1$ for $0 \leq t \leq \omega(X)$.

(3.10) Proposition. Let $X$ be a curve and let $\omega$ be any Whitney map for $C(X)$. If $X$ is a $\theta(2)$-curve and movable, then $\omega^{-1}(t)$ is also movable for $0 \leq t \leq$ $\omega(X)$ (see $[\mathbf{1}$ or $\mathbf{1 3}]$ for the definition of the movability).

PROOF. By $[8]$, the property of being pointed 1-movable is a Whitney property. By (3.9), $\mathrm{Fd} \omega^{-1}(t) \leq 1$ for $0 \leq t \leq \omega(X)$. Hence $\omega^{-1}(t)$ is movable (see $[\mathbf{1 3}, \mathrm{p}$. 199]).

It is well known that if $X$ is a continuum which is $\mathrm{Fd} X \leq 1$ and an FANR, then $\operatorname{Sh} X=\operatorname{Sh} \bigvee_{i=1}^{n} S_{i}$ for some $n<\infty$, where $\bigvee_{i=1}^{n} S_{i}$ denotes the one point union of $n$ circles.

(3.11) THEOREM. Let $X$ be a curve which is a $\theta(2)$-curve, and let $\omega$ be any Whitney map for $C(X)$. If $X$ is an FANR, $\omega^{-1}(t)$ is shape dominated by $X$, i.e., $\operatorname{Sh} \omega^{-1}(t) \leq \operatorname{Sh} X$. In particular, if $\operatorname{Sh} X=\operatorname{Sh} \bigvee_{i=1}^{n} S_{i}$, then $\operatorname{Sh} \omega^{-1}(t)=$ $\mathrm{Sh} \bigvee_{i=1}^{m} S_{i}$ for some $m \leq n$. Moreover, there is a positive number $t_{0}<\omega(X)$ such that $\operatorname{Sh} \omega^{-1}(t)=\operatorname{Sh} X$ for $0 \leq t \leq t_{0}$.

Proof. By (3.9) and (3.10), $\omega^{-1}(t)$ is movable and $\mathrm{Fd} \omega^{-1}(t) \leq 1$ for $0 \leq$ $t \leq \omega(X)$. Note that $\operatorname{Sh} \omega^{-1}(t)=\operatorname{Sh} \bigvee_{i=1}^{m} S_{i}$ for some $m=0,1,2, \ldots, \infty$. By [8, (1.13)], $H^{1}\left(\underline{f}_{0 t}\right): H^{1}\left(\omega^{-1}(t)\right) \rightarrow H^{1}(X)$ is a monomorphism. Since $\operatorname{Sh} X=$ Sh $\bigvee_{i=1}^{n} S_{i}(n<\infty)$, we conclude that $\operatorname{Sh} \omega^{-1}(t)=\operatorname{Sh} \bigvee_{i=1}^{m} S_{i}$ for some $m \leq n$. 
Hence $\omega^{-1}(t)$ is shape dominated by $X$. By $[8,(1.14)]$, there is a positive number $t_{0}<\omega(X)$ such that $H^{1}\left(\underline{f}_{0 t}\right)$ is an isomorphism for $0 \leq t \leq t_{0}$. This implies that $\operatorname{Sh} \omega^{-1}(t)=\operatorname{Sh} X$.

(3.12) EXAMPLE. In the statement of (3.11), we cannot omit the condition that $X$ is an FANR. Consider the set $X=\bigcup_{n=1}^{\infty} S_{n}$ in the plane $E^{2}$, where $S_{n}$ is the circle in $E^{2}$ with the center $((n-1) / n, 0)$ and raidus $1 / n$. Note that $X$ is a $\theta(2)$ curve and not an FANR. Let $\omega$ be any Whitney map for $C(X)$ and let $0<t<\omega(X)$. Then $\omega^{-1}(t)$ is an ANR such that $\omega^{-1}(t) \simeq \bigvee_{i=1}^{n} S_{i}$ for some $n<\infty$ (see [14]). Hence $\operatorname{Sh} X \neq \operatorname{Sh} \omega^{-1}(t)$ for $0<t \leq \omega(X)$.

4. Cell-like maps and Whitney continua. In $\S 3$, we proved that if $X$ is a strongly winding curve, then $\operatorname{Sh} \omega^{-1}(t)=\operatorname{Sh} X$ for any Whitney map $\omega$ for $C(X)$ and $0 \leq t<\omega(X)$. Note that if $X$ is a strongly winding curve, then each proper nondegenerate subcontinuum of $X$ is tree-like. Naturally, the following problem is raised: If $X$ is a curve and each proper nondegenerate subcontinuum of $X$ is tree-like, is $\operatorname{Sh} \omega^{-1}(t)=\operatorname{Sh} X$ for any Whitney map $\omega$ and $0 \leq t<\omega(X)$ ? In this section, by using the technique of Rogers [24, Theorem 3] and a theorem on cell-like map, we give a partial answer to the above problem.

A map $f: X \rightarrow Y$ from a compactum $X$ onto a compactum $Y$ is a cell-like map if $f^{-1}(y)$ has trivial shape for each $y \in Y$. It is well known that there is a cell-like map $f: X \rightarrow Y$ such that $\operatorname{Sh} X \neq \operatorname{Sh} Y, \operatorname{Fd} X<\infty$, and $\mathrm{Fd} Y=\infty$. We need the following (see [13, pp. 284 and 286]).

(4.1) Let $f: X \rightarrow Y$ be a cell-like map. If either (a) $\operatorname{Fd} X<\infty$ and $\operatorname{Fd} Y<\infty$ or (b) $\operatorname{dim} Y<\infty$, then $f$ is a shape equivalence.

(4.2) THEOREM. Let $X$ be a curve such that each proper nondegenerate subcontinuum of $X$ is tree-like. Assume that $\omega$ is a Whitney map for $C(X)$ and $\mathrm{Fd} \omega^{-1}(t)<\infty$ for some $t(0<t<\omega(X))$. Then the shape morphism $f_{0 t}: X \rightarrow$ $\omega^{-1}(t)$ is a shape equivalence, i.e., Sh $\omega^{-1}(t)=\operatorname{Sh} X$.

By (3.9) and (4.2), we have

(4.3) CoRollaRY. Let $X$ be a $\theta(m)$-curve $(m<\infty)$. If each proper subcontinuum of $X$ is tree-like, then $f_{0 t}: X \rightarrow \omega^{-1}(t)$ is a shape equivalence for any Whitney map $\omega$ for $C(X)$ and $0 \leq t<\omega(X)$, i.e., $\operatorname{Sh} \omega^{-1}(t)=\operatorname{Sh} X$.

Proof of (4.2). The proof is essentially due to J. T. Rogers [24, Theorem 3]. Consider the subset $M=\left\{(x, A) \mid x \in X, A \in C_{x}(X, \omega, t)\right\}$ of $X \times \omega^{-1}(t)$. Note that $M$ is a continuum. Let $p: M \rightarrow X$ and $q: M \rightarrow \omega^{-1}(t)$ be the projection maps. By (2.5) and the assumption, $p$ and $q$ are cell-like maps. Since $\operatorname{dim} X=1$, by (4.1) $p$ is a shape equivalence and $\mathrm{Fd} M \leq 1$. Also, by (4.1), $q$ is a shape equivalence. By the construction of $\underline{f}_{0 t}$, we can easily see that there exists a shape morphism $\underline{g}_{0 t}: X \rightarrow M$ such that $\underline{p} \cdot \underline{g}_{0 t}=\underline{1}_{X}$ and $\underline{q} \cdot \underline{g}_{0 t}=\underline{f}_{0 t}$. Since $\underline{p}$ and $\underline{q}$ are shape equivalences, we conclude that $\underline{f}_{0 t}: X \rightarrow \omega^{-1}(t)$ is a shape equivalence.

Finally, we give the following problem.

(4.4) Problem. Let $X$ be a curve. If each proper nondegenerate subcontinuum of $X$ is tree-like, is $\mathrm{Fd} \omega^{-1}(t) \leq 1$ for any Whitney map $\omega$ for $C(X)$ and $0<t<$ $\omega(X)$ ?

ADDED IN REMARK. Recently, the author proved that the problem (4.4) has an affirmative answer. 


\section{REFERENCES}

1. K. Borsuk, Theory of shape, Monograf. Mat. No. 59, PWN, Warszawa, 1975.

2. J. H. Case and R. E. Chamberlin, Characterization of tree-like continua, Pacific J. Math. 10 (1960), 73-84.

3. R. Duda, On the hyperspace of subcontinua of a finite graph. I, II, Fund. Math. 62 (1968), 265-286; 63 (1968), 225-255.

4. Correction to the paper On the hypersurface of subcontinua of a finite graph. I, Fund. Math. 69 (1970), 207-211.

5. D. Curtis, Stable points in hyperspaces of Peano continua, preprint.

6. J. T. Goodykoontz, Jr. and S. B. Nadler, Jr., Whitney levels in hypersurfaces of certain Peano continua, Trans. Amer. Math. Soc. 274 (1982), 671-694.

7. H. Kato, Concerning hyperspaces of certain Peano continua and strong regularity of Whitney maps, Pacific J. Math. 119 (1985), 159-167.

8. _ Shape properties of Whitney maps for hyperspaces, Trans. Amer. Math. Soc. 297 (1986), 529-546.

9. J. L. Kelley, Hyperspaces of a continuum, Trans. Amer. Math. Soc. 52 (1942), 22-36.

10. J. Krasinkiewicz, On the hyperspaces of snake-like and circle-like continua, Fund. Math. 83 (1974), 155-164.

11. _ Shape properties of hypersurfaces, Fund. Math. 101 (1978), 79-91.

12. J. Krasinkiewicz and S. B. Nadler, Jr., Whitney properties, Fund. Math. 98 (1978), 165-180.

13. S. Mardesić and J. Segal, Shape theory, North-Holland Mathematical Library, 1982.

14. M. Lynch, Whitney levels in $C_{p}(X)$ are absolute retracts, preprint.

15. S. B. Nadler, Jr., Hyperspaces of sets, Pure and Appl. Math., vol. 49, Dekker, New York, 1978.

16. _ Whitney-reversible properties, Fund. Math. 109 (1980), 235-248.

17. A. Petrus, Contractibility of Whitney continua in $C(X)$, General Topology Appl. 9 (1978), 275-288.

18. J. T. Rogers, The cone $=$ hypersurface property, Canad. J. Math. 24 (1972), 279-285.

19. __ Continua with cones homeomorphic to hyperspaces, General Topology Appl. 3 (1973), 283-289.

20. _ Dimension and Whitney subcontinua of $C(X)$, General Topology Appl. 6 (1976), 91-100.

21. __ Embedding the hypersurfaces of circle-like plane continua, Proc. Amer. Math. Soc. 29 (1971), 165-168.

22. __ Hyperspaces of arc-like and circle-like continua, Lecture Notes in Math., vol. 375, Springer-Verlag, Berlin and New York, 1974, pp. 231-234.

23. W Whitney continua in the hyperspaces $C(X)$, Pacific J. Math. 58 (1975), 569-584.

24. _ Applications of a Vietoris-Begle theorem for multi-valued maps to the cohomology of hyperspaces, Michigan Math. J. 22 (1975), 315-319.

25. J. Segal, Hyperspaces of the inverse limit space, Proc. Amer. Math. Soc. 10 (1959), 706709.

26. L. E. Ward, Jr., Extending Whitney maps, Pacific J. Math. 93 (1981), 465-469.

27. H. Whitney, Regular families of curves. I, Proc. Nat. Acad. Sci. U.S.A. 18 (1939), 184-192.

28. S. Nowak, Some properties of fundamental dimension, Fund. Math. 85 (1974), 211-227.

\section{Institute of Mathematics, UNIVERSity of TSUKUBA, IBARAKI, 305 JAPAN}

Current address: Faculty of Integrated Arts and Sciences, Hiroshima University, Higashisenda-Machi, Naka-Ku, Hiroshima 730, Japan 